Preprints of the

Max Planck Institute for

Research on Collective Goods

Bonn 2008/34

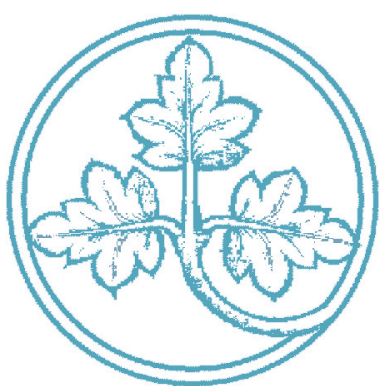

Playing strategically against nature?

- Decisions viewed from a game-theoretic frame

Martin Beckenkamp

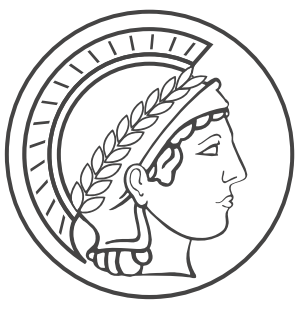




\section{Playing strategically against nature? \\ - Decisions viewed from a game-theoretic frame}

Martin Beckenkamp

September 2008 


\title{
Playing strategically against nature? \\ - Decisions viewed from a game-theoretic frame ${ }^{1}$
}

\author{
Martin Beckenkamp
}

\begin{abstract}
Common research on decision-making investigates non-interdependent situations, i.e., "games against nature". However, humans are social beings and many decisions are made in social settings, where they mutually influence each other, i.e., "strategic games". Mathematical game theory gives a benchmark for rational decisions in such situations. The strategic character makes psychological decision-making more complex by introducing the outcomes for others as an additional attribute of that situation; it also broadens the field for potential coordination and cooperation problems. From an evolutionary point of view, behavior in strategic situations was at a competitive edge. This paper demonstrates that even in games against nature, people sometimes decide as if they were in a strategic game; it outlines theoretical and empirical consequences of such a shift of the frame. It examines whether some irrationalities of human decision-making might be explained by such a shift in grasping the situation. It concludes that the mixed strategies in games against nature demand a high expertise and can only be found in situations where these strategies improve the effects of minimax-strategies that are used in cases of risk-aversion.
\end{abstract}

1 This paper has benefited from comments and suggestions received during presentations at the Max Planck Institute for Research on Collective Goods. I thank Arndt Bröder, who was a tremendous support for this paper. I also thank Ben Newell and Christoph Engel for very constructive suggestions, as well as Darrell Arnold and Brian Cooper for the helpful linguistic assistance. 


\section{Decision-Making Revisited: Individual and strategic decisions}

It is reasonable to assume that the evolution of human decision-making modules was triggered by competitive advantages resulting from mutual cooperation (cf., Tooby and Cosmides, 1992). However, common psychological research on decision-making investigates mainly situations no social interdependencies - the individual makes a decision on his own. Within this research, it has been common to distinguish two branches of decision theory. Descriptive decision theory is concerned with how decision-makers make decisions, whereas prescriptive decision theory develops normative concepts that identify optimal decisions that should be made in order to be rational. In both branches, decisions under certainty are distinguished from decisions under uncertainty.

The interest in problems under certainty in prescriptive decision theory was mainly triggered by complex problems; here, the complexity of the problem may lie in limitations of the cognitive capacity or in principal characteristics of the problem itself, such as the $N P$-completeness ${ }^{2}$ of a problem, or both. Given the NP-completeness of a problem, a general algorithmic solution for any problem-size becomes impossible; consequently, heuristic approaches have to be found if the problem itself is big enough. ${ }^{3}$ In descriptive decision theory, an important string of research is concerned with multi-attribute decision-making, although multi-attribute decisions under uncertainty also play an important role. Given the capacity limitations of cognitive processes, multi-attribute decision-making under certainty is a relevant issue. However, many of these models can also be applied to situations under uncertainty.

As already mentioned above, psychological literature about decision-making usually focuses on situations where the individual's decision is outside of a social context, i.e., the decision has no influence on the outcomes of other people, and others have no influence on the outcomes of the individual. Tagging the situation under uncertainty as a "game" in the sense of mathematical game theory, psychological decision-theory is usually concerned with "games against nature", where certain events happen independently of the individual's decisions (for instance if it rains or not), and the individual's outcome is determined by the event and the individual's decision for instance either the individual chose an umbrella or not. Subjective expected utility theory ( $S E U$ theory in the following) plays a prominent role within this context of games against nature. However, humans are social beings; and many decisions are made in social settings, where they mutually influence each other, i.e., humans often have to decide in "strategic games".

2 Easy, or tractable, problems can be solved by computer algorithms that run in polynomial time run time. The run time is not longer than a polynomial function of the problem size (like, for instance, number of objects to be sorted). np-complete problems, however, take more than polynomial time, i.e. with growing problem size the run-time increases severely. Many significant computer science problems belong to this category- e.g., the travelling salesman problem, or chess.

3 Indeed, the investigation of NP-complete problems was an important step on the path from traditional computer science to artificial intelligence, a predecessor discipline of cognitive science, and the investigation of heuristics is an important domain within artificial intelligence. 
Many of the situations that are grasped as games against nature could be grasped as strategic games as well. In the following it will be shown that such a shift in view has important consequences and may lead to different conclusions. Specifically, framing a situation as a social situation leads to the apposition of at least one more attribute, because besides the own outcome the consideration of the outcome of an additional person or a group comes along. As a result, many situations that are well-known in SEU theories may look different from the point of view of a strategic game. But this change is not a mere enrichment of the surface of the situation, but the theoretic solutions in such situations may differ as well. Therefore, it is also reasonable to assume that that a distinct view of the situation in the individual's mind should lead to different outcomes in their decision.

The following two sections will theoretically compare the classical $S E U$ view with the view of a strategic game. The fourth section introduces the concept of mixed strategies in strategic games with an empirical example (tennis). The fifth section reflects upon the consequences when mixed strategies that are adequate in strategic games are used in games against nature. The section is followed by an empirical example (section 6) that fits such a case where the mixed strategy solution describes well the behavior of the actors in a game against nature (offshore-fishery). This example was originally described as a clear case where $S E U$ is adequate. However, a subsequent theoretic analysis demonstrated that, even in games against nature, mixed strategies may help to overcome uncertainty. After these theoretic insights, the question is posed whether people apply mixed strategies in laboratory experiments about decisions against nature and where these strategies might make sense (section 7). The review suggests that mixed strategies are hard to learn; as a result, they cannot be observed in common laboratory experiments without a high repetition rate of the decision task.

\section{Decision-Making under Uncertainty: SEU Theories}

Typically, in decision theory the problem consists in making a choice between different alternatives. If the outcome for each alternative is known, the decision is made under certainty. If not, then the situation is made under non-certainty, or uncertainty in the broad sense. ${ }^{4}$ cases where the degree of uncertainty cannot be quantified (i.e., uncertainty in the narrow sense, also known as "Knightean uncertainty"). Decisions under risk refer to the quantifiable cases, i.e., cases where typically probabilities for the different outcomes are given or can be estimated. This distinction between certainty, uncertainty and risk corresponds to the traditional and prominent literature in decision-making (e.g., Luce \& Raiffa, 1957, p. 13) and is also used in the following sections.

Of course, many problems fall between the categories of risk and uncertainty, as defined by Luce and Raiffa (1957). Often, we do not know the precise probabilities, but nevertheless the probabilities are not completely unknown. It is also common to use the term "uncertainty" in such situations, and to name a situation under strict uncertainty a situation "under ignorance" (cf. Hansson, 2005, p. 27; Alexander, 1975, p. 365). Krizner (1994) introduces the case of unknown problem spaces. For the purpose of clarity, this terminology and these distinctions will not be used in the following sections. The following arguments concentrate on the two poles of uncertainty with, on the one hand, Knightean uncertainty and, on the other, uncertainty with given probabilities. 
The major paradigms for decisions under risk are expected value theory ( $E V$ theory) and $S E U$ theory (subjective expected utility theory). According to $E V$ theory, the alternative with the greatest $E V$ should be chosen. The expected value of a choice $X$ is calculated with:

$$
E(X)=\sum_{i=1}^{n} p_{i} x_{i}
$$

The expected value of a choice $X$ is calculated by multiplying the probabilities for different outcomes in nature $p_{i}$ by the resulting payoff in this state of the nature $x_{i}$. Considering either subjective probabilities for a subject $j$ (instead of objective probabilities) or utilities (instead of objective outcomes), or both, extends the scope to $S E U$ theory (cf. Savage 1954):

$$
\operatorname{SEU}(X)=\sum_{i=1}^{n} p_{i j} u_{j}\left(x_{i}\right)
$$

Given an option $\mathrm{X}$ and $n$ different possible outcomes in nature, $S E U$ is calculated by multiplying the utilities for subject $j u_{j}\left(x_{i}\right)$ by the subjective probabilities $p_{i j}$. Again, the decision-maker is assumed to choose an option $X$, with the highest expected utility.

However, it is important to note that the probabilities are "fixed", i.e., the respective probabilities of the outcomes are independent of the subject's decision. This is what characterizes "games against nature": A lottery is a good example of a game against nature. In contrast, in strategic games a chosen alternative may influence others, and the choices of others have impacts on one's own outcome: recent examples of bookmaking demonstrated that customers were in the belief to play a game against nature (like a lottery), whereas in fact there was a manipulation and therefore they were playing a strategic game. Endogenous (instead of exogenous) uncertainty arises in strategic games, where the outcome depends on social interaction (cf. Heinemann, Nagel \& Ockenfels 2004). This aspect is usually not considered in the relevant literature on psychological decision-making, as will be demonstrated with the following example.

\section{Strategic Decision-Making Under Uncertainty}

In a text book about decision-making (Jungermann, Pfister \& Fischer, pp. 201-214), the following example of one application of $S E U$ theories can be found. A grandson is not sure about the testament of his grandfather, and he does not know whether he will inherit a lot of money from him. Depending on whether he will be rich (if he inherits the money) or not (if he does not inherit the money), the consequences of living in Hamburg (a city that is expensive, but with very high quality of life) or Dortmund (a city that is cheap, but with moderate quality of life) will be different. They are given in the following matrix. As it is common in decision theories, the different states of nature (i.e., the situation in which either the grandson will inherit the money and be rich or the one in which he will not inherit the money and be poor) are given in the columns. The different options of the grandson (moving to Hamburg or staying in Dortmund) are given in the row. The outcomes of the different decisions with corresponding utilities of the grandson are inscribed in the cells of the matrix (cf. table 1): 


$$
E_{1} \text { Inherits money } \quad E_{2} \text { Does not inherit money }
$$

\begin{tabular}{lll}
\hline X Hamburg & $\mathrm{x}_{1}$ (rich in Hamburg) & $\mathrm{x}_{2}$ (poor in Hamburg) \\
& $u\left(\mathrm{x}_{1}\right)=1.0$ & $u\left(\mathrm{x}_{2}\right)=0.0$ \\
Y Dortmund & $\mathrm{y}_{1}($ rich in Dortmund) & $\mathrm{y}_{2}$ (poor in Dortmund) \\
& $u\left(\mathrm{y}_{1}\right)=0.7$ & $u\left(\mathrm{y}_{2}\right)=0.3$ \\
\hline
\end{tabular}

This example is discussed as a game against nature, and - according to SEU theory - the choice should be made that maximizes the expected utility of a choice:

$$
\max \left(\sum_{i=1}^{n} p_{i j} u_{j}\left(x_{i}\right), \sum_{i=1}^{n} p_{i j} u_{j}\left(y_{i}\right)\right)
$$

In the example, the grandson can either choose $X$ or $Y$; and according to $S E U$, he should choose that option that maximizes his expected utility. In the given situation the grandson should live in Hamburg as long as the probability to inherit the money is greater than $1 / 2$, and live in Dortmund if the probability is below that. The probability $p=1 / 2$ for both events $E_{1}$ and $E_{2}$ provides the point of indifference between the two choices, because $1 / 2(0.7)+1 / 2(0.3=1 / 2(1)+1 / 2(0)=0.5$. If the probability of inheriting the money is above $1 / 2$, the grandson should choose Hamburg; otherwise, he should choose Dortmund. The grandson could also be risk averse and try to maximize his minimal payoffs by using a maximin strategy: maximize the minimal gain. In this case, independently of the probabilities of the different outcomes, the grandson would stay in Dortmund, ensuring him a minimal utility of 0.3 .

At first glance, these seem to be rather convincing approaches, but it could well be that the grandson thinks that he is in a strategic situation, such that the probability of inheriting the money depends on whether he stays near his grandfather (i.e., he chooses Dortmund) or whether he moves (i.e., to Hamburg). For instance, it could be that his grandfather will become angry if the grandson moves to Hamburg, and the probability of getting the inheritance will be lower than if the grandson stays in Dortmund. This strategic view of the situation introduces a second attribute; namely, the payoffs of his grandfather become relevant. The grandson also has to consider his grandfather's utilities in the matrix. In other words, looking at a decision situation from the point of view of a strategic game can be understood as changing it from a one-attribute decision-making situation to a two-attribute decision-making situation. Let's name the corresponding utilities for the grandfather $v$. The corresponding matrix might be depicted as follows: 


\begin{tabular}{lll}
\hline X Hamburg & $\mathrm{x} 1$ (rich in Hamburg) & $\mathrm{x} 2$ (poor in Hamburg) \\
& $\mathrm{u}(\mathrm{x} 1)=1.0$ & $\mathrm{u}(\mathrm{x} 2)=0.0$ \\
& $\mathrm{v}(\mathrm{x} 1)=0.0$ & $\mathrm{v}(\mathrm{x} 2)=0.1$ \\
Y Dortmund & $\mathrm{y} 1$ (rich in Dortmund) & $\mathrm{y} 2$ (poor in Dortmund) \\
& $\mathrm{u}(\mathrm{y} 1)=0.7$ & $\mathrm{u}(\mathrm{y} 2)=0,3$ \\
& $\mathrm{v}(\mathrm{y} 1)=1.0$ & $\mathrm{u}(\mathrm{y} 2)=0.7$ \\
\hline
\end{tabular}

In strategic games, it also often makes a difference whether the decisions are made sequentially or simultaneously. In a simultaneous game, the grandson could take the risk and choose $\mathrm{X},{ }^{5}$ whereas he should not choose $\mathrm{X}$ as a first mover, because in this case, his grandfather would maximize his utility by choosing $\mathrm{E}_{2}$, ending up with a utility $\mathrm{v}\left(\mathrm{x}_{2}\right)=0.1$, which is better for him than $\mathrm{v}\left(\mathrm{x}_{1}\right)=0.0$. Therefore, in a sequential game, the grandson should choose Dortmund. This would lead to a utility of 0.7 for him and 1.0 for his grandfather.

\section{Mixed Strategies}

But what about the situation when both decisions are made simultaneously and are irreversible? In this case, a mixed strategy solution may be useful. Although it can be calculated for strategic situations as in the example provided before, for the sake of simplicity let us first consider zeroor constant-sum games, i.e., games where the benefit of one actor corresponds to a loss of the other such that the sum of all players' outcomes is the same in any of the possible cases (i.e., cells of the matrix): The gain of the proponent entails the loss of the opponent, and vice versa. In this case, the matrix of outcomes reduces in complexity, because the indication of one of the two actors' outcomes and the indication of the constant sum is sufficient for the representation of the situation. ${ }^{6}$ A tennis game can serve as an example of such a situation, where the server has to decide whether to play to the forehand or the backhand of the opponent. The opponent decides whether he should expect to be served to the forehand side or the backhand side. The opponent has a better chance of a good return if he expects the ball on the side the server is serving to. However, the backhand is usually weaker than the forehand, and this is why the chance that the server will serve to the backhand is higher than the chance that he will serve to the forehand. The following matrix could characterize such a situation (cf. table 3 ).

5 However, this is not an equilibrium solution. The mixed strategy solution for this game that makes the Grandfather indifferent between E1 and E2, is to choose Hamburg with $\mathrm{p}=0.75$. The calculus for such solutions will be introduced below.

6 This makes constant-sum games look similar to games against nature, but still it is a strategic situation where the actor has to consider that the other reacts on what he chooses. An explanation follows below. 
Table 3

Table of successful attack/defense rates in a tennis game.

Expect forehand

Expect backhand

Serve forehand

0.2

0.4

0.8

0.6

Serve backhand

0.6

0.3

0.4

0.7

As mentioned above, this matrix is redundant and one value per cell (instead of two) would be sufficient, because the success rate of the proponent is 1- the failing rate of the opponent (this is precisely the characteristic of zero-sum games). Nevertheless, it is not an SEU situation, but a strategic one. The solution for the server is to play such that the opponent becomes indifferent about expecting a serve to the forehand as opposed to the backhand. Any other strategy would raise the success rate of the opponent (and decrease the success rate of the server). Naming the probability that a ball will be served to the forehand side $p(S f)$ and the probability that a ball will be served to the backhand side $p(S b)=1-p(S f)$, the indifference point (and equilibrium) $E q$ is found by calculating the following: ${ }^{7}$

$E q=p(S f) 0.2+(1-p(S f)) 0.6=p(S f) 0.4+(1-p(S f)) 0.3$

The solution of this equation is 0.6 , meaning that the server should serve forehand with a probability 0.6. The value of that game, i.e., the success rate for the server, is 0.36 if he uses that strategy.

Walker and Wooders (2001) applied the MSE theory (Mixed strategy equilibrium theory) to data from professional tennis matches. The serve in tennis can be described as a 2x2 mixed-strategy game. Walker and Wooders obtained data from matches between world-class tennis players, who should be expert in the strategic subtleties of this game. The way they play corresponds quite closely to the mixed-strategy predictions. Thus, the empirical evidence to date indicates that mixed strategy predictions may be effective in explaining and predicting behavior in strategic situations when the competitors are experts, and that it is less effective when the competitors are novices (cf. Walker \& Wooders, 2001 and 2006).

\section{Mixed Strategies in Games Against Nature}

Now, taking the situation in table 3, let us compare the results of a minimax-strategy with the result of MSE. In cases where one and the same situation is repeated many times, by making use of a mixed strategy, the success rate increases from 0.30 - the solution of a pure minimax-

7 A comprehensible explanation for the derivation of that formula can be found in Straffin (1993). 
strategy - to 0.36 : In equation 4 , we calculated that a probability mix of 0.6 for serving to the forehand $(p(S f)=0.6)$ and 0.4 for serving to the backhand $(p(S b)=0.4)$ makes the opponent indifferent as to whether he expects that a forehand will be served or expecting that a backhand will be served. According to a pure minimax strategy, the server would always serve to the backhand (and the opponent would ideally always expect that). In this case, the "payoff", or success-rate, for the server is 0.3 (cf. table 3 ). However, by choosing the calculated probability mix, the expected "payoff" of the server is at least 0.36 , independent of the behavior of the opponent. ${ }^{8}$

This insight leads to interesting consequences with respect to games against nature: Even in games against nature it can make sense to use mixed strategies, although games against nature are not strategic situations. It makes sense if an individual is risk averse and in a situation of (Knightean) uncertainty, i.e., where the probabilities for different outcomes in nature are not known, and where either the situation is repeated for many times or the choices can be split up in accordance with the mixed-strategy solution (like for instance in portfolios, where one can put $36 \%$ in $\mathrm{A}$ and $64 \%$ in B). Therefore, the mixed-strategy solution may be a good improvement of a minimax-strategy in games against nature.

Above that, in situations with Knightean uncertainty, it would be impossible to apply $S E U$ (instead of minimax). By making use of the mixed-strategy solution, the actor decides on the (implicit) presumption that nature would try to fool him and be completely against him. Although the presumption sounds ridiculous, the decision itself is not at all! This way of making use of a mixed strategy can be grasped as a maximin strategy under complete uncertainty, and the application leads to an insensitivity about the wrong estimations of risks. In other words: the opponent (or nature ${ }^{9}$ ) may do what he/it wants; the actor can (still) be guaranteed not to fall under the expected value of the mixed strategy ( 0.36 in the strategic tennis case above). Of course, this is not true for a single trial, but as already mentioned above it concerns the expected result in the long run, or the case when a decision can be split up simultaneously in many sub-decisions.

\section{Empirical evidence for mixed strategies in games against nature}

In the literature, there is also some empirical evidence for the use of such a strategy in a game against nature, which implicitly leads to optimized risk-averse behavior under the assumption of complete uncertainty. In an anthropological study, Davenport (1960) observed that fishing crews adopted their fishing strategies very near to the game-theoretic mixed-strategy solution. The fishing grounds were divided into inside and outside banks, and the captains of the canoes could adopt three different fishing strategies: inside (put all pots on the inside banks), outside (put all pots on the outside banks) and in-out (put some pots on the inside banks and some pots on the outside). The yields for the three different strategies depend on whether dangerous currents come

8 This can be calculated by putting the calculated probability mix in equation 4 and calculating the value: $E q=0.6 * 0.2 * 0.4 * 0.6=0.6 * 0.4+0.4 * 0.3=0.36$

9 So far, we have considered the situation of a strategic game. The illustration fort his point in a game against nauture follows below. 
up or not (cf. table 4). According to Davenport's game-theoretic analysis in mixed strategies, it is optimal (in mixed strategies) to have 67\% inside and 33\% in-out. Actually, 69\% followed the inside strategy and $31 \%$ the in-out strategy. Please note that in this example it is possible to split the choices, and therefore the mixed strategy will always guarantee the minimal gain that is calculated in the mixed-strategy solution.

Table 4

The payoff table for the fishermen according to Davenport (1960)/Straffin (1993).

\begin{tabular}{llcc} 
& & \multicolumn{2}{c}{ Current } \\
\cline { 3 - 4 } & & Run & Not Run \\
\hline \multirow{3}{*}{ Fishermen } & Inside & 17.3 & 11.5 \\
& Outside & -4.4 & 20.6 \\
& In-Out & 5.2 & 17.0 \\
\hline
\end{tabular}

But why should the fishermen make use of the mixed-strategy solution? The probabilities that dangerous currents may come up are well known, with a $25 \%$ chance of a "run" and a $75 \%$ chance of "no run". Therefore, it is also possible to calculate a solution with SEU theory. According to this solution, the expected value would be maximized if all fishermen fish outside. It would be 14.35. The expected value in case of mixed strategies is only 13.3, independent of the current's probability to run.

In this example, a further advantage of the mixed-strategy solution in games against nature becomes obvious. Compared to mixed strategies, $S E U$ is very risky: on days when the currents run and all fishermen are outside as the SEU suggests, the fishermen will suffer losses. If losses loom larger than gains, or if people are risk-averse respectively, it is better to make use of the mixed-strategy solution. Risky choices are choices that tolerate a higher variance in the outcomes and correspondingly one cannot be sure about one's concrete outcome. By making use of mixed strategies in this example the security level of 13.3 is guaranteed in any case. ${ }^{10}$

Let us summarize some insights with respect to decision-making: Whereas SEU may maximize expected payoffs, a mixed strategy is an insurance against errors in the estimates of the probabilities. It is the safe harbor and even optimal in scenarios with complete uncertainty. Therefore, making use of mixed strategies may help to avoid dropping below important thresholds, as we will see in the following example about financial assets. ${ }^{11}$

10 Readers who are interested in the calculation of different solutions in Davenport's example should compare the excellent and lucid presentation in Straffin, 1993 (pp. 23-27).

11 Again, please note that the thresholds are expected values and the outcome of random processes, such that in short time spans the strategy may fall below the threshold, but in the long run this becomes more and more unlikely. 
The considerations given so far are not merely theoretical, but they have consequences in the consideration of common $S E U$ strategies. The following example demonstrates that it may make sense to apply corresponding portfolio strategies, where "nature" is an efficient market. Let us assume that we have a sum of money that we can divide up into two different investments: in an annuity fund and in a risky fund. The expected payoffs for the upcoming investment period will depend on the developments of the market, either with growth or stagnation (cf. table 5).

Table 5

A payoff table for investment decisions.

\begin{tabular}{lcc} 
& E1: Stagnation & E2: Growth \\
\hline$X$ (Bonds) & 15 & 14 \\
$Y$ (risky funds) & -5 & 40 \\
\hline
\end{tabular}

According to a pure minimax strategy, in this situation one should choose $\mathrm{X}$, which provides a security level of 14 units. However, by making use of a mixed strategy, this security level can be raised up to 14.56 units. Solving the following equation provides the solution:

$15 p_{X}-5 p_{Y}=14 p_{X}+40 p_{Y} \rightarrow 15 p_{X}-5\left(1-p_{X}\right)=14 p_{X}+40\left(1-p_{X}\right)$

It yields $p=45 / 46$, such that, according to a mixed strategy, $97.83 \%$ of the assets should be invested in bonds. ${ }^{12}$ The secure gain for the investor from this strategy is about 14.57. According to $S E U$, the following term should be maximized:

$\max \left(15 p_{\mathrm{O} 1}+14 p_{\mathrm{O} 2},-5 p_{\mathrm{O} 1}+40 p_{\mathrm{O} 2}\right)$

There are also some formal relations between the mixed-strategy solution and solutions found in $S E U$. The solution of the mixed strategy shows the point at which the investor making use of $S E U$ should change his investment from the bonds to the risky funds: i.e., according to $S E U$, if $p_{\mathrm{O} 1}<0.4348$, then the investor should choose the risky option.

However, it does not always make sense to make use of mixed strategies in such situations against nature. A graphic representation of the situation reveals an easy way to see whether making use of a mixed strategy makes sense in a given situation (cf. figure 1). We plot the different events of nature on the abscissa, the payoffs on the ordinate, and represent the choices in different lines. Given such a graphic representation of the decision situation it only makes sense to use a mixed strategy if the lines cross between the different outcomes and if one of the lines has a positive slope and the other line has a negative slope (cf. figure 1). Otherwise it makes sense to use pure strategies, because in this case either one strategy dominates another or the risk-averse

12 According to this calculation, nature "optimizes" if $p \mathrm{E} 1=20 / 46$, such that in $43.48 \%$ of the cases, it should choose E1. However, as it is a game against nature, the interpretation of this result hardly makes sense. 
solution is the pure minimax solution, - or both (i.e., when there is no crossing and both lines have the same positive/negative slope).

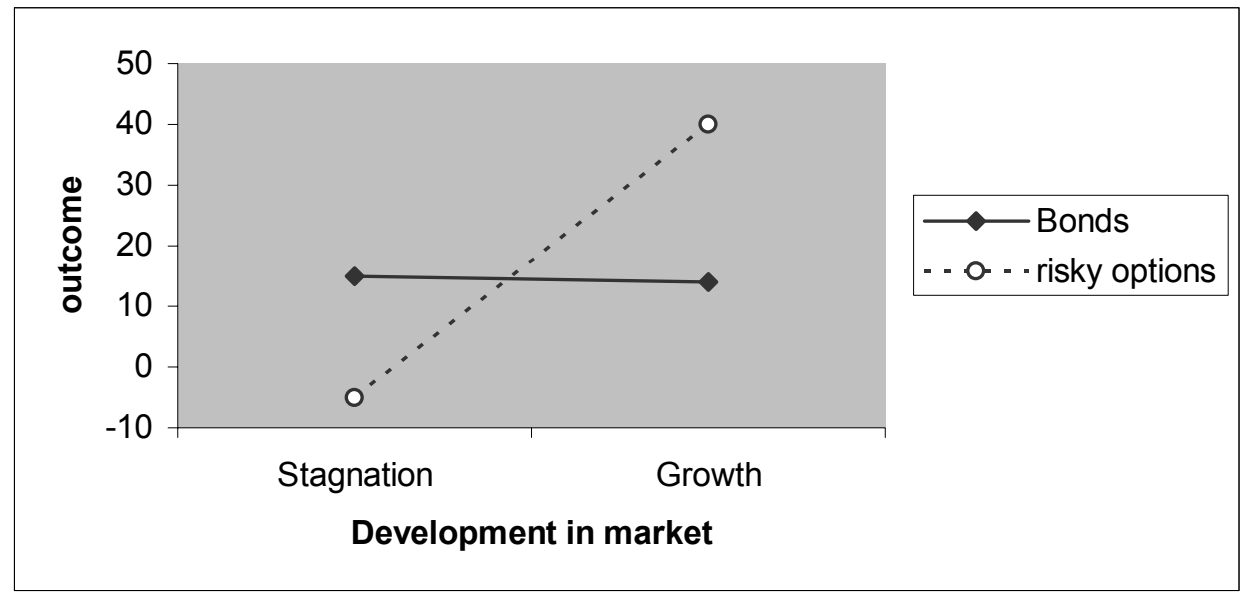

Figure 1. A graphical representation of the decision situation in table 5.

To summarize so far: It is worth thinking about mixed strategies as decision routines aiming to overcome uncertainty, even in situations against nature. From a historian's point of view, this might even be obvious: traditionally, many cultures played strategically with nature - they quarreled with their gods. It seems rather that "nature" is a modern invention that replaced strategic decision-making with and against the gods, who arranged one's fate (cf. Snell 1953).

\section{Experimental Data Revisited: Do People Apply mixed Strategies in Games against Nature?}

The arguments given so far provide good theoretic reasons for why playing strategically against nature can make sense and may even be rational in cases of Knightean uncertainty. However, besides the Davenport example, is it plausible to assume that such strategies can often be empirically found in decision-making contexts? There are already a number of experimental designs on decision-making in which it would make sense to make use of mixed strategies, although the experiments were not conceived for this purpose. One class of such experiments are experiments on probability matching. Revisiting such results might help to answer the empirical question about the use of mixed strategies in games against nature. Typically, in such experiments about probability matching, subjects have to choose between two options, for instance, red and green. After their decision, an outcome is determined randomly. The experiment is repeated for many rounds, and the probabilities of the outcomes are held constant. In case of a match between the choice and the outcome, the subject receives a payoff (cf. table 6). 
A symmetric payoff table used in probability-matching experiments.

IS RED

5

(WR)

WANT GREEN

(WG)
IS GREEN

0

According to rational choice (and $S E U$ ), if the payoff matrix is symmetric with respect to the two different errors and hits, subjects should choose that option that has a higher probability to occur. Only in cases where the probabilities (and the payoffs) for both outcomes are equal can subjects choose whatever they want. However, it is a typical empirical result that many subjects fail at this task, and one common error is that subjects engage in probability-matching instead; i.e., if the probability for red is 0.9 and the probability for green is 0.1 , then subjects' rates of choices of red and green correspond to these probabilities instead of always choosing red. The use of strategies is dependent on the task, like whether balls are drawn from an urn or whether a die is rolled (cf. Gal \& Baron 1996). On a global level (taking both tasks together), Gal and Baron found that about $68 \%$ of the subjects' answers were correct (they always chose red); about $20 \%$ almost always chose red but they sometimes chose green (although green was chosen with a relative frequency lower than 0.1 ); about $10 \%$ chose the matching strategy; and about $2 \%$ chose half-half. The main purpose of their study was to re-examine people's understanding of optimality in such repeated simple choices. The argument in the paper at hand enriches their re-examination, providing an additional reason that has not been brought up in discussions about this issue so far: although people may deliberatively reason that it is ridiculous to assume that urns or dies behave strategically, they might at least have such a fear and behave correspondingly. ${ }^{13}$

Schul, Mayo, Burnstein and Yahalom (2007, p. 91) demonstrate that people use different strategies in dependence of these situations: "We assume that when they have to predict outcomes that are attributed to non-human causes, people acknowledge their ignorance and try to focus on what is most diagnostic. However, when events are attributed to human agency, they believe that nothing is arbitrary and that one can understand the decision situation well enough to eliminate error. If so, then people should behave differently when an uncertainty is attributed to chance (a nonhuman agency) or to deception (a human agency)." In their experiments, they find that individuals who attribute uncertainty to deception (instead of attributing it to chance) are less likely to adopt the optimal rule-based strategy. However, they overlook the fact that, in cases of decep-

13 There are full of examples with confusions of games against nature with strategic games (and vice versa), such as the belief that a curse may have a negative impact on others. Magical thinking is a well-known phenomenon both in anthropology, clinical psychology and developmental psychology. The examples given so far suggest that some people may have such magical thinking in the context of rolling a dice or drawing lots from an urn, but also go beyond that by demonstrating that in some situations there is a lot of rationality in such magical thinking. 
tion, minimax provides a strategy that guarantees a security level. Their argument is that, under fear of deception, individuals attempt to understand their interaction partners so that they can decipher their strategies (Schul et al., 2007, p. 95). In contrast to this point and under the point of view of mixed strategies, it could also well be that people experience a feeling of not really being able to decipher the strategies of others. Nevertheless, they can improve their results and can be less vulnerable by making themselves both unpredictable and by considering their own payoffs and the payoffs of the opponent.

So, at first glance, a strategy like probability matching might reflect the idea that subjects make use of mixed strategies in this case. With this view, in cases of identical payoffs for the two different hits and identical payoffs for the two different errors that may occur in the decision situation, the mixed-strategy solution is always to choose both options with a probability of $1 / 2$, independent of the knowledge about base rates. Asymmetric choice rates depend on asymmetries in the payoffs, i.e., if the two different hits and/or the two different errors have different payoffs. Therefore, the much more severe deviation that we would expect from a mixed-strategy point of view can be observed in experimental data on probability matching in asymmetric payoff matrices (cf. table 7).

Table 7

An asymmetric payoff table used in probability-matching experiments. The numbers in brackets give the presumed payoffs of the opponent, if the situation is seen as a zero-sum game.

IS LEFT

$2,(-2)$

$(\mathrm{WL})$

WANT RIGHT

(WR)
IS RIGHT

$0,(0)$

$1,(-1)$
$0,(0)$

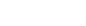

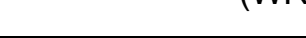

In this decision situation, subjects were asked to play the following virtual matrix game (in the role of the row player) against an anonymous opponent. $63 \%$ of the subjects $(n=2029)$ chose WL and $37 \%$ WR. However, the mixed-strategy solution (independent of the actual rates of L and R) is to choose WL with probability $1 / 3$ (cf. Rubinstein, 2006, p. 5). In this case, the opponent (nature) is indifferent about playing LEFT or RIGHT:

$$
E q=p(W L) 2+p(W R) 0=p(W L) 0+p(W R) 1 \Rightarrow p(W L)=1 / 3 ; p(W R)=2 / 3
$$

Choosing $1 / 3$ WL and $2 / 3$ WR would save an expected earning of $2 / 3$ units per trial in the long run. However, subjects deviate completely from that strategy.

Now a puzzle emerges: Why do the data in Rubinstein (2006) and many other experiments about probability matching not correspond to mixed strategies, whereas the data from Davenport (1960) on fishery and from Walker and Wooders (2006) about world-class tennis players fit so 
well? It seems that learning is relevant, because typically in repeated mixed-strategy games, it can be found that people learn adequate strategy mixes. This contrasts with the results about probability matching. Walker and Wooders (2006, p. 4) conclude: "Thus, the empirical evidence to date indicates that MSE [i.e., mixed-strategy equilibrium M.B.] is effective for explaining and predicting behavior in strategic situations at which the competitors are experts and that it is less effective when the competitors are novices, as experimental subjects typically are." These findings suggest that the acquisition of adequate probability mixes in mixed strategies is a consequence of other learning mechanisms and not a consequence of a predefined machinery or module in our minds for such purposes.

\section{Conclusion}

In the context of multi-attribute decision-making, it seems at least worthwhile to take a closer look at the social intelligence of homo sapiens and to ask both empirically and theoretically whether human evolution adapted decision modules to social situations. Comparing mere individual decisions with decisions in social situations introduces at least one more attribute into the process of decision-making, because it is not only one's own outcomes that matter. Instead, the outcomes of others also have to be considered - both from a theoretic point of view, by shifting from SEU theories to game-theoretic concepts, and from an empirical point of view. In this paper, the question has been raised whether it might be even possible that, when subjects are in a situation of individual decision-making, they still decide as if they were in such a social context. A consequence of such "biases" is that instead of maximizing the expected value, prudent and security-seeking behavior may be adapted, which always takes the worst outcomes into account. Mixed strategies generalize such ideas of pure minimax stategies in $S E U$ theories and perform (slightly) better. Until now, this theoretic point has been overlooked in psychological research on decision-making under uncertainty.

However, a closer look at empirical data shows that individuals do not coherently use mixed strategies. Empirical examples with experts can be found in field research, but empirical results from the lab in the context of probability-matching stand in obvious contradiction to this strategic idea. Therefore, there results indicate that individuals and communities adapt to mixed strategies after long experience. Such adaptations seem to take place even in games against nature, which theoretically makes sense under the point of view of risk aversion. Bringing to mind that the concept of nature as a pure random process is a rather new invention in cultural history, compared to the quarrel with gods who intentionally influence our destiny, it seems plausible that people often make use of minimax-strategies. It could well be that people adapted to mixed strategies due to the evolutionary pressure, without knowledge about probabilities and probability mix. $S E U$ would have predicted that people adapt to strategies that maximize the expected values, whereas mixed strategies would predict an adaption to strategies that guarantee rather constant results that reduce the given uncertainty of the situation. 
Therefore, it seems plausible that mixed strategies can be rational even in games against nature. But when is it rational and when not? Humans are social beings and are able to co-operate. In the context of co-operation, it may be advantageous to use strategies that make the individual predictable. In situations of competition, mixed strategies that create unpredictability make sense. Humans have learned both: to cooperate and to cheat.

This might also explain why animals perform better in probability-matching tasks than humans do (cf. Schul et al., 2007). The authors do also allude to the higher costs of the attempts to understand contingencies as strategic interactions instead of just making use of statistic reasoning. The consideration and review on experiments given in my paper have demonstrated that indeed it is hard to learn mixed strategies. However, the considerations given here contradict their conclusions that it is the absence of immediate reliable feedback that makes it difficult to learn by statistic reasoning. The authors claim that statistical reasoning would be more useful for dealing with uncertainty than to understand the situation as a strategic interaction. In contradiction to this conclusion, here it has been demonstrated that the use of mixed strategies in probabilitymatching experiments is very useful, but that it is hard to learn. The paper at hand suggests that the social world triggers the learning of elements of mixed strategies, and that experts in competitive settings (like match games) may also be experts in the use of such strategies. However, due to the difficulties and long-lasting learning processes, it seems implausible to assume that there is a mental tool that directly corresponds to the calculation of mixed strategies. Erev and Roth (1998) demonstrate in simulations that reinforcement learning may lead to an adequate mixed strategy. Assuming that mixed strategies are learned via the reinforcement-learning module, it is obvious that it takes a long time to learn to come close to the mixed strategy solution, and that the use of mixed strategies is associated with expertise. 


\section{References}

Alexander, E. R. (1975). The limits of uncertainty: A note. Theory and Decision, 6, 363-370.

Davenport, W. H. (1960). Jamaican fishing: A game theory analysis. Papers in Caribbean Anthropology, 59, 3-11.

Erev, I., \& Roth, A. E. (1998). Predicting how people play games: reinforcement learning in experimental games with unique mixed strategy equilibria. American Economic Review, $88(4), 848-881$.

Gal, I., \& Baron, J. (1996). Understanding Repeated Simple Choices. Thinking and Reasoning, 2, 81-98.

Hansson , S. O. (2005). Decision theory: A brief introduction. Retrieved December 19, 2006, from http://www.infra.kth.se/ $\sim$ soh/decisiontheory.pdf.

Heinemann, Nagel, R., \& Ockenfels, P. (2004). Measuring strategic uncertainty in coordination games. Retrieved March 6, 2007, from

http://www.wm.tu-berlin.de/ makro/Heinemann/download/HNO-2.pdf.

Jungermann, H., Pfister, H.-R., \& Fischer, K. (1998). Die Psychologie der Entscheidung. Heidelberg: Spektrum.

Knight, F. H. (1921). Risk, Uncertainty and Profit. Boston, MA: Hart, Schaffner \& Marx; Houghton Mifflin Company.

Krizner, I. (1994). On the economics of time and ignorance. The market process. In P. J. Boettke \& D. L. Prychitko (Eds.), The Market Process. Essays in Contemporary Austrian Economics (pp. 38-44). Brookfield: Elgar.

Luce, R. D., \& Raiffa, H. (1957). Games and Decisions. New York: Wiley.

Rubinstein, A. (2006). Instinctive and Cognitive Reasoning: A Study of Response Times. Retrieved December 19, 2006, from http://cess.nyu.edu/0012:2004-03.pdf.

Savage, L. J. (1954). The Foundations of Statistics. New York: Wiley.

Schul, Y., Mayo, R., Burnstein, E., \& Yahalom, N. (2007). How people cope with uncertainty due to chance or deception. Journal of Experimental Social Psychology, 43, 91-103.

Snell, B. (1953). The discovery of the mind. Oxford: Blackwell.

Straffin, P. D. (1993). Game theory and strategy. Washington: The Mathematical Association of America. 
Todd, P. M., Fiddick, L., \& Krauss, S. (2000). Ecological rationality and its contents. Thinking and Reasoning, 6(4), 375-384.

Tooby, J., \& Cosmides, L. (1992). The psychological foundations of culture. In J. Barkow, L. Cosmides \& J. Tooby (Eds.), The adapted mind: Evolutionary psychology and the generation of culture (pp. 19-136). New York: Oxford University Press.

Walker, M., \& Wooders, J. (2001). Minimax play at Wimbledon. American Economic Review, 91, 1521-1538.

Walker, M., \& Wooders, J. (2006). Mixed strategy equilibrium. Retrieved December 19, 2006, from http://econ.arizona.edu/downloads/working papers/Econ-WP-06-04.pdf. 